\title{
Arthritis of the base of the thumb
}

\author{
Carrie R. Swigart
}

Published online: 24 February 2008

(C) Humana Press 2008

\begin{abstract}
The purpose of this article is to outline the pathophysiology and epidemiology of arthritis of the base of the thumb. The usual presentation and diagnosis will be discussed along with the current conservative treatment options. Surgical treatment options are determined by the stage of the arthritis as well as the demands of the patient. The current standard surgical treatment options will be reviewed along with their results in the literature.
\end{abstract}

Keywords Arthritis - Thumb - Basal joint - CMC joint

\section{Introduction}

The base of the thumb, primarily the trapeziometacarpal (TM) joint, is the second most common site affected by primary idiopathic arthritis in the hand behind only the distal joints of the fingers. Symptomatic arthritis in this area can cause a much more significant functional disability, however, due to the broad range of activities that become impaired. It is important to remember the unique nature of the human thumb basal joint. During the evolution of our thumbs, the TM joint became biconcave allowing motion in three planes and allowing for true opposition. This change, along with the development of muscles to provide active opposition, increased the importance of the ligaments that stabilize the joint during

C. R. Swigart $(\bowtie)$

Department of Orthopaedics and Rehabilitation, Yale University

School of Medicine, New Haven, CT, USA

e-mail: carrie.swigart@yale.edu pinch. Acute injury or gradual incompetence of these ligaments can result in joint degeneration.

\section{Pathophysiology}

While both biochemical and biomechanical factors play a role in the development of arthritis at the base of the thumb, it is the biomechanical design of the joint that makes it unique. In order to allow for the tremendous degree of motion at the TM joint there is very little intrinsic osseous stability. This puts considerable strain on the supporting ligaments even during routine daily activities. If these ligaments become unable to stabilize the joint it is felt that joint degeneration will follow in a predictable fashion [1-3]. The joint initially becomes painful due to synovitis. As the disease progresses, there is a loss of articular cartilage, first in the palmar regions of the joint and spreading to involve the entire joint. Osteophytes form in a futile attempt to increase the surface area and thereby decrease the force per unit area. Lastly, in end stage disease, the thumb metacarpal base will subside and a thumb adduction contracture will occur.

\section{Epidemiology}

Approximately one in four women and one in twelve men will develop radiographic evidence of joint degeneration although not all will become symptomatic. Women with symptomatic involvement of the base of the thumb outnumber men by a ratio of 10-15:1 [4]. Racial differences have also been observed, especially between Asian and White populations. Postmortem studies in whites show a $50 \%$ rate of severe arthritis at the TM joint [5], whereas 
similar studies in the Japanese literature document an $8 \%$ rate of severe joint changes [6].

\section{Presentation and diagnosis}

Patients with arthritis of the basal joint will typically complain of pain at the base of the thumb particularly with pinch grasp activities such as opening jars or bottles and turning keys. Needlecrafts such as sewing and knitting will become painful. Hobbies such as gardening and many racquet sports will often cause a worsening of the pain. On physical examination palpation directly over the thumb base or TM joint will cause pain. With advanced arthritis, motion of this joint will often elicit crepitance.

Radiographs of the thumb should be obtained although the degree of symptom severity does not correlate with the amount of radiographic joint involvement. In order to best visualize the basal joint area of the thumb, a 30-degree oblique view of the hand is useful (Fig. 1). The joint laxity of early stage disease can often be demonstrated with a "basal joint stress" view (Fig. 2). Late stage arthritis will often show complete loss of the joint space, subchondral cysts, osteophytes, and involvement of the scaphotrapezial joint (Fig. 3). The degree of arthritis seen on the radiograph will determine the available treatment options, but should not be the sole determinate of any decision regarding surgery.

During the evaluation of patients complaining of pain at the base of the thumb it is important to eliminate other causes of thumb pain such as deQuervain's disease, thumb triggering and metacarpophalangeal arthritis. The patient should also be evaluated for possible carpal tunnel syndrome that has been found to coexist in up to $43 \%$ of patients with basal joint arthritis [7].

\section{Conservative treatment}

Non-steroidal anti-inflammatories (NSAIDs)

As for most arthropathies, NSAIDs can often be quite effective and should be used as tolerated in the early treatment of symptoms. Their use should be tailored to the symptom pattern of the individual patient and do not generally need to be given on a long-term basis.

\section{Splinting}

Initial treatment of thumb basal joint arthritis also relies on reducing inflammation by resting the joint. The patient has usually self modified their activities to certain extent because of the pain. The TM joint can be further rested by immobilizing it in a splint. Custom molded splints fabricated by a

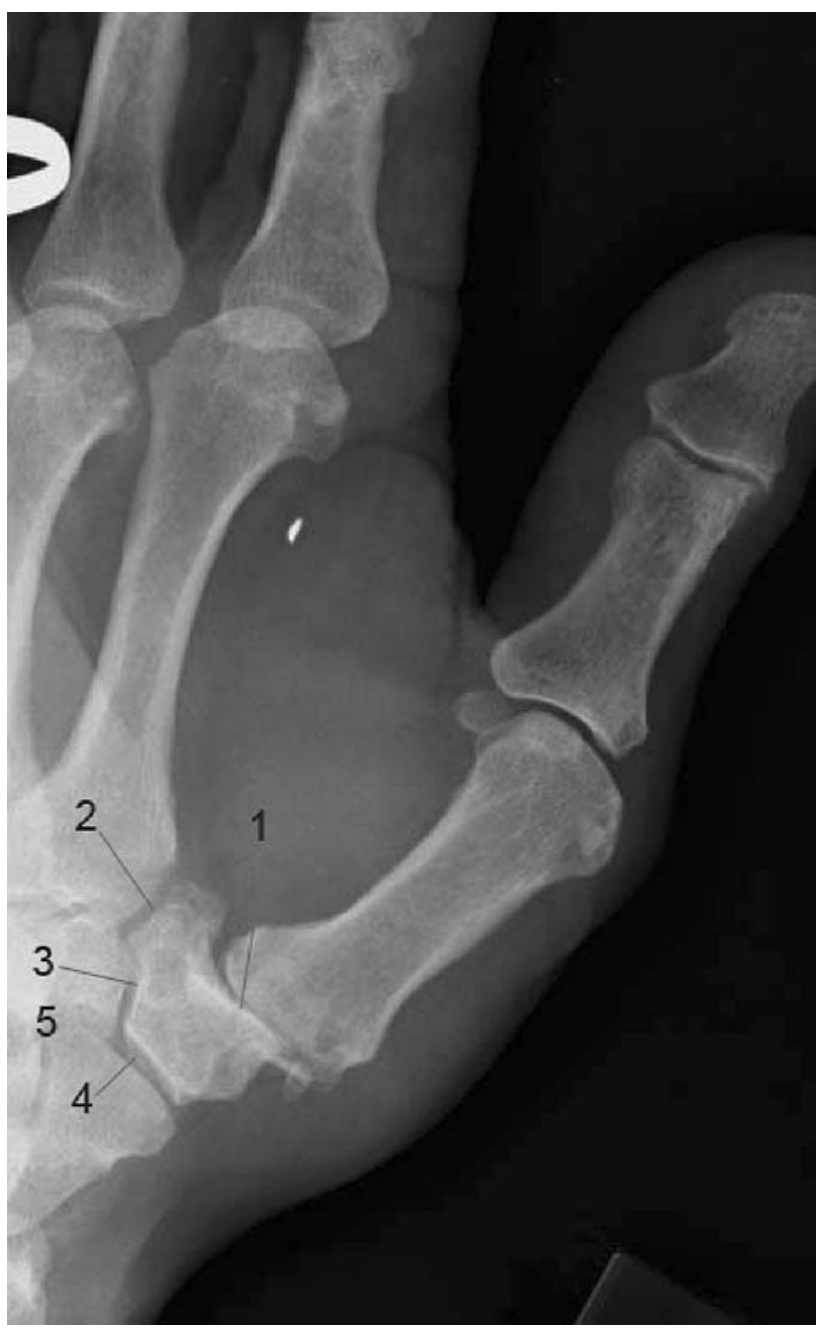

Fig. 1 A radiograph of the thumb base taken at a 30 degree oblique angle showing the five articulations of the "basal joint". 1trapeziometacarpal joint (thumb), 2-trapezium-index metacarpal, 3-trapezium-trapezoid, 4-scaphotrapezial, 5-scaphotrapezoidal

knowledgeable hand therapist are the most comfortable and therefore the most effective. Use of a long thumb splint (Fig. 4) to immobilize the wrist and thumb for 6 weeks has been found to have a greater degree of symptom relief in early stage disease as compared to later stage disease, but is recommended as an initial treatment for all patients [8]. A shorter splint that allows freedom of wrist motion may be better tolerated by some patients and can be effective in resting the TM joint as well (Fig. 5). In one recent study of 33 patients followed over 7 years hand therapy and splinting helped $70 \%$ avoid surgery altogether [9].

\section{Hand therapy}

Therapy for early stage disease may also include thenar muscle strengthening and education in how to modify pinch-grasp activities, so as to load the joint without undue 


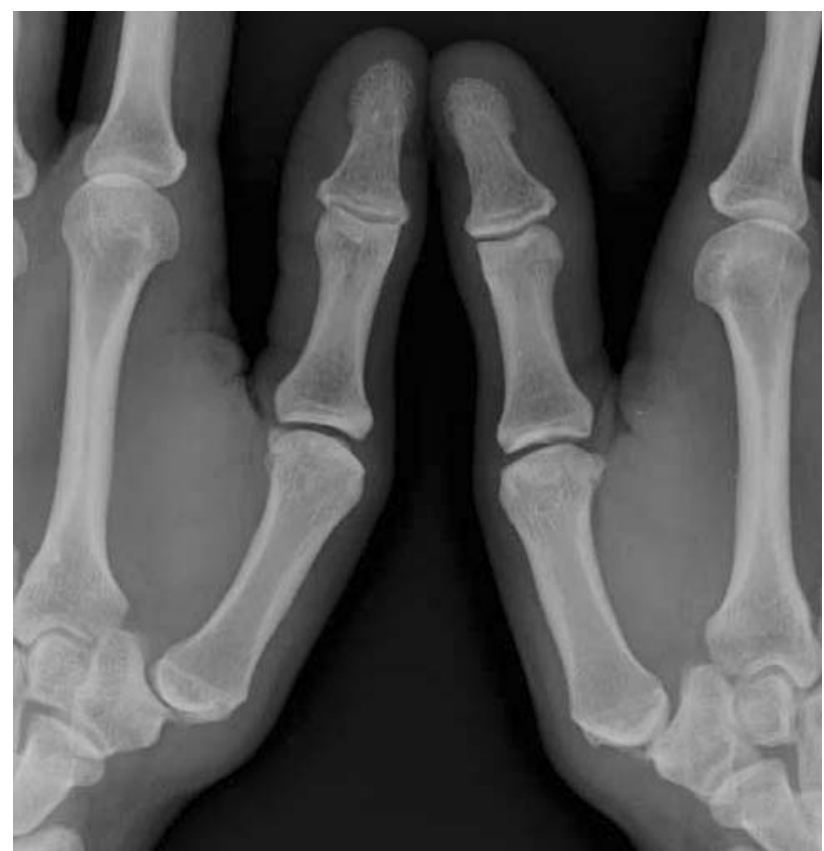

Fig. 2 A "stress" radiograph of the thumbs showing early stage arthritis with subluxation of the thumb metacarpal base on the trapezium

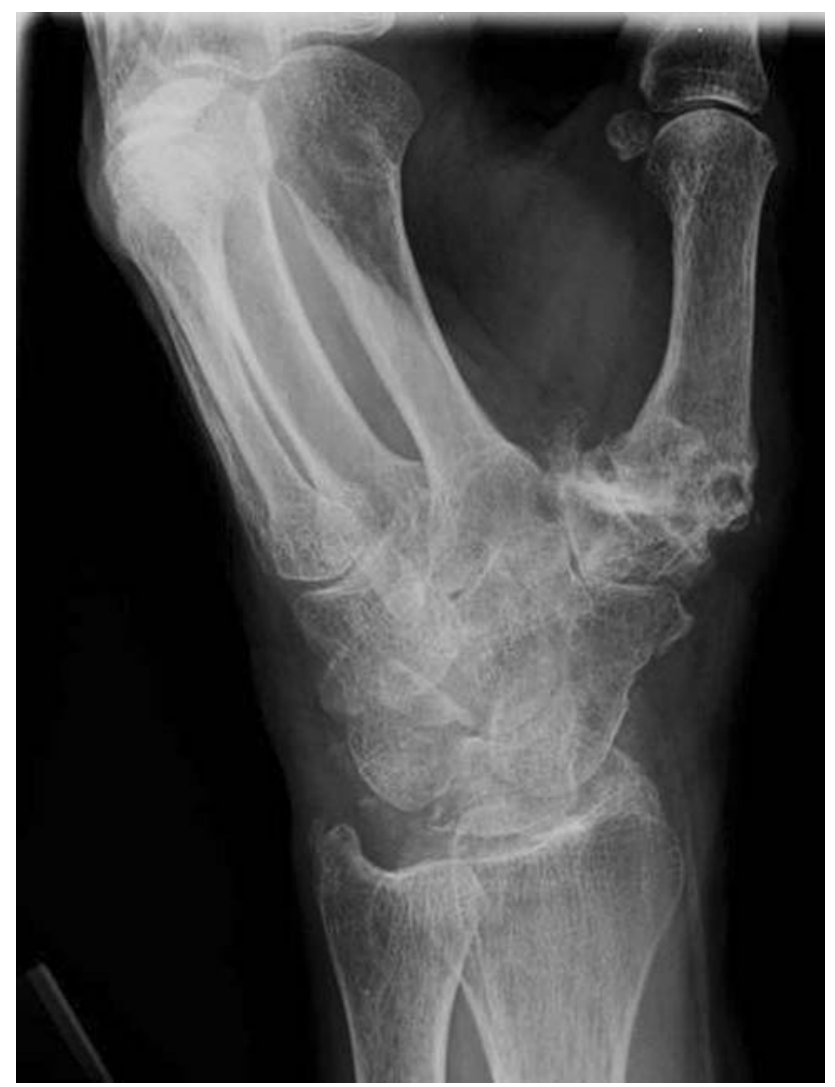

Fig. 3 Late stage arthritis of the basal joint involving all of the articulations
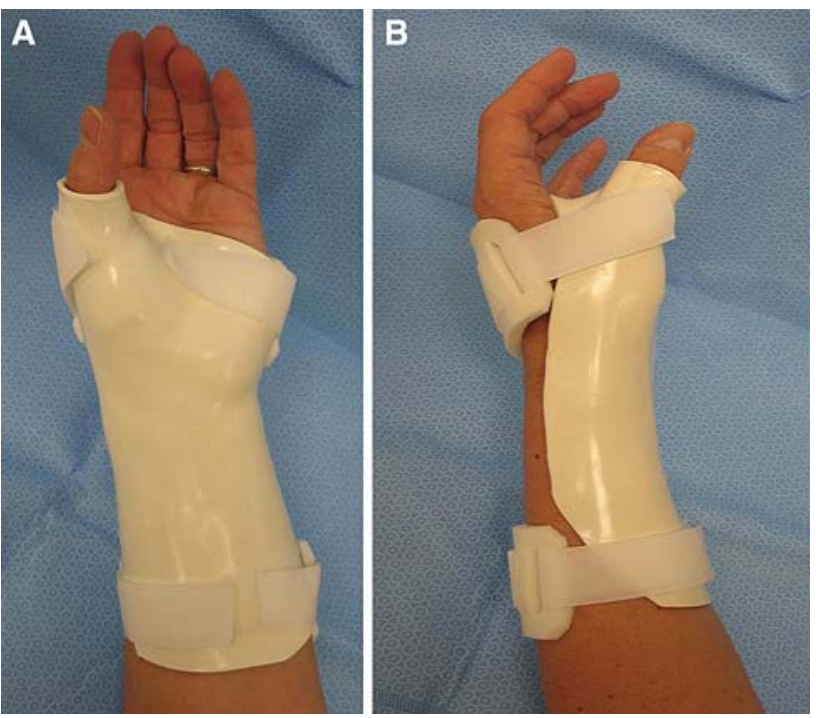

Fig. 4 (a, b) Orthogonal views of a custom molded long thumb splint
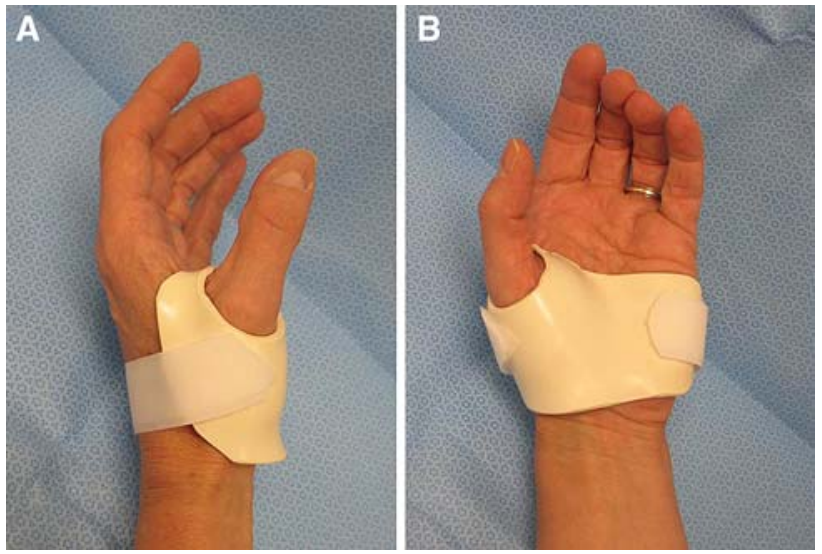

Fig. 5 (a, b) Orthogonal views of a custom molded short thumb splint with the thumb IP joint left free

shear stress. Modalities such as heat and massage may offer temporary relief of symptoms. Aside from the fabrication of custom molded splints, the hand therapist has little to offer patients with late stage arthritis.

\section{Corticosteroid injection}

Injection of corticosteroid into the TM joint can be used in early to moderate stage disease. The effect of injection is usually temporary with both the degree of symptom relief and the duration of effect being quite variable. In patients with only inflammation and no radiographic signs of joint changes the effects can be long term, whereas patients with late stage arthritis tend to obtain no significant long-term relief from injection [10]. 


\section{Surgical treatment}

When conservative treatment of thumb basal joint arthritis fails, there are numerous surgical alternatives. Surgical indications include pain, deformity, and/or weakness that interferes significantly in daily hand functions. The choice of which surgery best suites the individual is based upon both the radiographic stage of disease and the activity requirements of the patient. In broad terms, the procedures can be divided into two groups-those for early stage arthritis and those for late stage arthritis.

In patients for whom there is only early cartilage softening and fibrillation but with no actual cartilage loss or eburnation, symptoms are usually as a result of joint laxity and resultant inflammation. Surgical treatment focuses on the creation of a stable joint by reconstruction of the palmar beak ligament. In order to accomplish this, a slip of flexor carpi radialis (FCR) tendon is used as described by Eaton and Littler [11]. The FCR slip is left attached to its insertion on the index metacarpal base and passed through a bone tunnel created in the base of the thumb metacarpal and back around to be sutured to itself again. This reconstruction of the palmar beak ligament has been shown to provide good to excellent results in early stage arthritis [12], and has more recently been shown to prevent progression of arthritis in two-thirds of patients [13].

Osteotomy of the thumb metacarpal was originally used in late stage disease in order to correct a fixed thumb adduction deformity. More recently is has been shown to yield good results for early stage arthritis [14]. The osteotomy is designed to extend the metacarpal approximately by 30 degrees and thereby reduce the joint reaction force in the palmar compartment where the first signs of cartilage loss usually appear [15].

For later stage arthritis in young patients with high physical demands of the hand, arthrodesis (or fusion) may be the best alternative. A successful arthrodesis will provide a stable and pain-free thumb for forceful grasp and pinch activities. The loss of thumb CMC motion that results is usually well tolerated. Comparison of arthrodesis and arthroplasty has shown similar outcomes in range of motion, strength, pain relief, and patient satisfaction [16]. The methods of fixation vary from simple smooth pins to more rigid plates and screws. Rates of non-union have been reported from 7 to $10 \%$ and complications have been found to be higher when plates and screws were used to secure the fusion [17].

By far, the most common procedure performed for moderate to late stage disease is some form of soft-tissue arthroplasty. This includes trapezial excision alone, trapezial excision with soft-tissue interposition, and trapezial excision with reconstruction of the palmar beak ligament (with or without interposition of soft-tissue into the trapezial space). Simple excision of the trapezium was described in 1949 and has been used -as a relatively easy surgery for both the surgeon and patient [18]. Although originally described without any soft-tissue interposition, some surgeons add the interposition of material such as the palmaris longus tendon. This has been done in an attempt to prevent subsidence of the thumb metacarpal base, but a recent study has shown no difference in outcomes between trapezial excision alone and with additional soft-tissue interposition [19].

Regardless of any interposition material used, subsidence of the thumb metacarpal will occur after trapeziectomy. However, unless there is actual impingement of the metacarpal on the scaphoid, the degree of proximal migration of the thumb has not been correlated with outcome [20]. Nonetheless, the most common softtissue arthroplasty used today includes a reconstruction of the palmar beak ligament with either all or half of the FCR tendon as well as an interposition of the remaining tendon material into the trapezial space, commonly referred to as the LRTI (ligament reconstruction tendon interposition). The method of ligament reconstruction is similar to that used for early stage arthritis. There are five series in the literature that report patient outcomes after LRTI [21-25]. Pain relief and patient satisfaction have been shown in $85 \%$ of patients. Grip strength typically returns more quickly and fully compared to key pinch, although pinch strength can improve for 6 years after surgery. The degree of thumb metacarpal subsidence has never been shown to correlate with results of strength or pain relief.

Finally, there are a variety of implant arthroplasties. Silicone implant arthroplasty became popular in the 1970s. It has since fallen out of favor due primarily to problems with implant wear and resultant silicone particulate synovitis and osteolysis. It is currently recommended only in the low demand patient with inflammatory arthritis [26]. Hemiarthroplasty implants replacing only the TM joint have been fabricated out of silicone and metal. Silicone implants have been abandoned due to the above mention problems. A stemmed titanium implant that is placed into the thumb MC base has been available since 1985, but no long-term data has been reported. A spherical implant developed as a spacer for the TM joint has shown disappointing early results with complications including dislocation, trapiezial fracture or erosion, and severe pain. Total joint arthroplasty has been used for the TM joint in the form of a cemented ball and socket type implant. The original design, introduced by la Caffiniere in 1971, is used and marketed today primarily in Europe [27]. Although there is a total joint prosthesis marketed in the US today, most studies still show variable results with higher overall complication rates. 


\section{Conclusion}

Pain at the base of the thumb due to inflammation and/or degeneration of the basal joint(s) is a common problem. It will affect 10-15 times as many women as men, primarily in the fifth and sixth decades of life. Conservative treatment consists primarily of splinting and NSAIDs, regardless of the radiographic stage of the arthritis. Those patients with more advanced arthritic degeneration are more likely to fail conservative treatments. When conservative treatments fail to provide sufficient relief, the choice of surgical procedure depends on the stage of joint degeneration and the physical demands of the patient.

\section{References}

1. Pellegrini VD. Osteoarthritis of the thumb trapeziometacarpal joint: a study of the pathophysiology of articular cartilage degeneration: I. anatomy and pathology of the aging joint. J Hand Surg [Am]. 1991;16:967-74.

2. Pellegrini VD. Osteoarthritis of the thumb trapeziometacarpal joint: a study of the pathophysiology of articular cartilage degeneration: II. articular wear patterns in the osteoarthritic joint. J Hand Surg [Am]. 1991;16:975-82.

3. Jonnson H, et al. Hypermobility associated with osteoarthritis of the thumb base: a clinical and radiological subset of hand osteoarthritis. Ann Rheum Dis. 1996;55a:540-43.

4. Kellgren J, Moore R. Generalized osteoarthritis and nodes. BMJ. 1952;1:181-7.

5. Cooney WP III, Chao EY. Biomechanical analysis of static forces in the thumb during hand function. J Bone Joint Surg Am. 1977;59:27.

6. Fujisawa K. Arthrosis of the carpometacarpal joint of the thumb (the third report): a comparative radiographic and anatomical study. J Jpn Soc Surg Hand. 1988;5:412-5.

7. Florack TM, Miller RJ, Pellegrini VD, et al. The prevalence of carpal tunnel syndrome in patients with basal joint arthritis of the thumb. J Hand Surg [Am]. 1992;17A:624-30.

8. Swigart CR, Eaton RG, Glickel SZ, Johnson C. Splinting in the treatment of arthritis of the first carpometacarpal joint. J Hand Surg [Am]. 1999;24A:86-91.

9. Berggren M, Joost-Davidsson A, Lindstrand J, et al. Reduction in the need for operation after conservative treatment of osteoarthritis of the first carpometacarpal joint: a seven year prospective study. Scand J Plast Reconstr Surg Hand Surg. 2001;35:415-7.

10. Day CS, et al. Basal joint osteoarthritis of the thumb: a prospective trial of steroid injection and splinting. J Hand Surg [Am]. 2004;29A:247-51.
11. Eaton RG, Littler JW. Ligament reconstruction for the painful thumb carpometacarpal joint. $\mathrm{J}$ Bone Joint Surg Am. 1973;55:1655-66.

12. Lane LB, Eaton RG. Ligament reconstruction for the painful "prearthritic" thumb carpometacarpal joint using a modified Eaton and Littler technique. Clin Orthop. 1987;220:52-7.

13. Freedman DM, Eaton RG, Glickel SZ. Long-term results of volar ligament reconstruction for symptomatic basal joint laxity. J Hand Surg [Am]. 2000;25:297-304.

14. Tomaino MM. Treatment of Eaton stage I trapeziometacarpal disease with thumb extension osteotomy. J Hand Surg [Am]. 2000;25:1100-6.

15. Pellegrini VD, Parentis M, Judkins A, et al. Extension metacarpal osteotomy in the treatment of trapeziometacarpal osteoarthritis: a biomechanical study. J Hand Surg [Am]. 1996;21:16-23.

16. Hartigan BJ, Stern PJ, Kiefhaber TR. Thumb carpometarcarpal osteoarthritis: arthrodisis compared with ligament reconstruction and tendon interposition. J Bone Joint Surg [Am]. 2001;83:1470-8.

17. Forseth MJ, Stern PJ. Complication of trapeziometacarpal arthrodesis using plate and screw fixation. J Hand Surg [Am]. 2003;28:342-5.

18. Gervis WH. Excision of the trapezium for osteoarthritis of the trapeziometacarpal joint. J Bone Joint Surg [Br]. 1949;31:537-9.

19. Davis TRC, et al. Excision of the trapezium for osteoarthritis of the trapeziometacarpal joint: a study of the benefit of ligament reconstruction or tendon interposition. J Hand Surg [Am]. 2004;29:1069-77.

20. Lins RE, Gelberman RH, McKeown L, et al. Basal joint arthritis: trapeziectomy with ligament reconstruction and tendon interposition arthroplasty. J Hand Surg. 1996;21:202-9.

21. Lins RE, Gelberman RH, McKeown L, et al. Basal joint arthritis: trapeziectomy with ligament reconstruction and tendon interposition arthroplasty. J Hand Surg. 1996;21:202-9.

22. Nylen S, Johnson A, Rosenquist A-M. Trapeziectomy and ligament reconstruction for osteoarthrosis of the base of the thumb. J Hand Surg [Br]. 1993;18:616-9.

23. Rayan GM, Young BT. Ligament reconstruction arthroplasty for trapeziometacarpal arthrosis. J Hand Surg [Am]. 1997;22:1067-76.

24. Tomaino MM, Pellegrini VD, Burton RI. Arthroplasty of the basal joint of the thumb: long-term follow up after ligament reconstruction with tendon interposition. J Bone Joint Surg Am. 1995; 77:346-55.

25. Varitimidis SE, Fox RJ, King JA, et al. Trapeziometacarpal arthroplasty using the entire flexor carpi radialis tendon. Clin Orthop. 2000;370:164-70.

26. Pellegrini VD Jr, Burton RI. Surgical management of basal joint arthritis of the thumb: I. long-term results of silicone implant arthroplasty. J Hand Surg [Am]. 1986;11:309.

27. de la Caffiniere JY, Aucouturier P. Trapeziometacarpal arthroplasty by total prosthesis. Hand. 1979;11:41-6. 